\title{
On approximation of two-dimensional potential and singular operators
}

\author{
Charyyar Ashyralyyev and Sedanur Efe \\ Communicated by Vladimir Shaydurov
}

\begin{abstract}
The purpose of this paper is the construction of second-order of accuracy quadrature formulas for the numerical calculation of the Vekua types two-dimensional potential and singular integral operators in the unit disk of complex plane. We propose quadrature formulas for these integrals which based on first-order spline approximation of twodimensional function. MATLAB programs are used for numerical experiments in test examples.
\end{abstract}

Keywords. Spline approximation, potential integral, singular integral, quadrature formula, numeric integrals.

2010 Mathematics Subject Classification. 65D30, 65E05, 45P05, 30E20.

\section{Introduction}

Two-dimensional potential and singular integrals in the circle of complex plane are used to solve widely range of models in applied mechanics and various branches of physics (see [1-13] and references therein).

In paper [3], the first-order of accuracy quadrature formulas for the numerical calculation of the following two-dimensional potential and singular integral operators in the unit disk of complex plane

$$
T(\rho \mid z)=-\frac{1}{\pi} \iint_{K} \frac{\rho(\zeta)}{\zeta-z} d \zeta, S(\rho \mid z)=-\frac{1}{\pi} \iint_{K} \frac{\rho(\zeta)}{(\zeta-z)^{2}} d \zeta, z \in K
$$

where $K=\{z \in C:|z| \leq 1\}$ are considered. These operators were introduced by I. Vekua [13]. Some properties of operators $T(\rho \mid z)$ and $S(\rho \mid z)$ are described in $[10$, p. 198-210]. In particular,

$$
\frac{\partial T(\rho \mid z)}{\partial z}=S(\rho \mid z), \frac{\partial T(\rho \mid z)}{\partial \bar{z}}=\rho(z), z \in K
$$


are given. Our purpose in this paper is the construction of second-order of accuracy quadrature formulas for the numerical calculation of the operators $T(\rho \mid z)$ and $S(\rho \mid z)$.

Following [3], we will use grid sets for the unit disk $K$ in the complex plane. First, we introduce the following notations:

$$
\begin{aligned}
& {[0,1]_{\tau}=\left\{r_{k} \mid r_{k}=k \tau, 1 \leq k \leq N, N \tau=1\right\},} \\
& {[-\pi, \pi]_{h_{k}}=\left\{\theta_{k, m} \mid \theta_{k, m}=-\pi+m h_{k}, 0 \leq m \leq M_{k},\right.} \\
& \left.M_{k} h_{k}=2 \pi, M_{k}=2 k+1,1 \leq k \leq N\right\} ; K_{\tau}=\{z \in C:|z|<\tau\}, \\
& K_{r_{k}}=\left\{z \in C:|z|<r_{k}\right\}, 1 \leq k \leq N ; H_{r_{k}}=K_{r_{k+1}}-K_{r_{k}} .
\end{aligned}
$$

These notations permit us to write

$$
K=K_{\tau} \bigcup \bigcup_{k=1}^{N-1} H_{r_{k}}=K_{\tau} \bigcup \bigcup_{k=1}^{N-1} \bigcup_{m=0}^{M_{k}-1} D_{k, m}
$$

where domain $D_{k, m}$ is defined by

$$
\begin{gathered}
D_{k, m}=\left\{\zeta \mid \zeta=r e^{i \theta}, r_{k} \leq r \leq r_{k+1}, 1 \leq k \leq N-1,\right. \\
\left.\theta_{k, m} \leq \theta \leq \theta_{k, m+1}, 0 \leq m \leq M_{k}-1\right\}
\end{gathered}
$$

with boundary $\partial D_{k, m}$ :

$$
\begin{aligned}
& \partial D_{k, m}=\Gamma_{k m}=\Gamma_{k m}^{1} \cup \Gamma_{k m}^{2} \cup \Gamma_{k m}^{3} \cup \Gamma_{k m}^{4}, \\
& \Gamma_{k m}^{1}=\left\{\zeta \mid \zeta=r e^{i \theta_{k, m+1}}, r_{k} \leq r \leq r_{k+1}\right\}, \\
& \Gamma_{k m}^{2}=\left\{\zeta \mid \zeta=r_{k+1} e^{i \theta}, \theta_{k, m+1} \geq \theta \geq \theta_{k, m}\right\}, \\
& \Gamma_{k m}^{3}=\left\{\zeta \mid \zeta=r e^{i \theta_{k, m}}, r_{k+1} \geq r \geq r_{k}\right\}, \\
& \Gamma_{k m}^{4}=\left\{\zeta \mid \zeta=r_{k} e^{i \theta}, \theta_{k, m} \leq \theta \leq \theta_{k, m+1}\right\}
\end{aligned}
$$

and its four corner points are

$$
\begin{aligned}
& z_{0,0}^{k, m}=r_{k} e^{i \theta_{k, m}}, z_{1,0}^{k, m}=r_{k+1} e^{i \theta_{k, m}}, \\
& z_{1,1}^{k, m}=r_{k+1} e^{i \theta_{k, m+1}}, z_{0,1}^{k, m}=r_{k} e^{i \theta_{k, m+1}}
\end{aligned}
$$

for any $0 \leq m \leq M_{k}, 1 \leq k \leq N$.

Second, we introduce grid points

$$
\begin{gathered}
z_{0,0}^{* k, m}=\left(r_{k}+\frac{\tau}{2}\right) e^{i\left(\theta_{k, m}+\frac{h_{k}}{2}\right)}, z_{1,0}^{* k, m}=\left(r_{k+1}+\frac{\tau}{2}\right) e^{i\left(\theta_{k, m}+\frac{h_{k}}{2}\right)}, \\
z_{1,1}^{* k, m}=\left(r_{k+1}+\frac{\tau}{2}\right) e^{i\left(\theta_{k, m+1}+\frac{h_{k}}{2}\right)}, z_{0,1}^{* k, m}=\left(r_{k}+\frac{\tau}{2}\right) e^{i\left(\theta_{k, m+1}+\frac{h_{k}}{2}\right)}
\end{gathered}
$$


in which the approximate values of $T$ and $S$ will be calculated and denote by $D_{k, m}^{*}$ corresponding small domains with four corner points. Thus, we have two sets of gridpoints $\Omega^{(1)}$ and $\Omega^{(2)}$ defined by

$$
\begin{aligned}
& \Omega^{(1)}=\Omega_{\tau, h}^{(1)} \\
& =\left\{z_{0,0}^{k, m}, z_{1,0}^{k, m}, z_{1,1}^{k, m}, z_{0,1}^{k, m} \mid 1 \leq k \leq N-1,0 \leq m \leq M_{k}-1\right\}, \\
& \Omega^{(2)}=\Omega_{\tau, h}^{(2)} \\
& =\left\{z_{0,0}^{* k, m}, z_{1,0}^{* k, m}, z_{1,1}^{* k, m}, z_{0,1}^{* k, m} \mid 1 \leq k \leq N-1,0 \leq m \leq M_{k}-1\right\} .
\end{aligned}
$$

\section{Spline approximation}

In this section, we describe approximation of complex valued function $\rho$ by the first-order splines in two variables $z$ and $\bar{z}$. From $z=x+i y, \bar{z}=x-i y$, it follows $x=\frac{z+\bar{z}}{2}, y=\frac{z-\bar{z}}{2 i}$. So, applying first-order two dimensional splines in variables $x$ and $y$, we have the following approximation (see [2, p.12, formula (1.16)]):

$$
\rho(z) \approx \widehat{\rho}(z)=a_{k m} z+b_{k m} \bar{z}+c_{k m}\left(z^{2}-\bar{z}^{2}\right)+d_{k m}, z \in D_{k, m},
$$

where unknown coefficients $a_{k m}, b_{k m}, c_{k m}, d_{k m}$ are defined by given values of $\rho$ at four corner points (2). Hence, we get linear system equations

$$
\begin{aligned}
& \rho\left(z_{0,0}^{k, m}\right)=a_{k m} z_{0,0}^{k, m}+b_{k m} \overline{z_{0,0}^{k, m}}+c_{k m}\left[\left(z_{0,0}^{k, m}\right)^{2}-{\overline{z_{0,0}^{k, m}}}^{2}\right]+d_{k m}, \\
& \rho\left(z_{1,0}^{k, m}\right)=a_{k m} z_{1,0}^{k, m}+b_{k m} \overline{z_{1,0}^{k, m}}+c_{k m}\left[\left(z_{1,0}^{k, m}\right)^{2}-{\overline{z_{1,0}^{k, m}}}^{2}\right]+d_{k m}, \\
& \rho\left(z_{1,1}^{k, m}\right)=a_{k m} z_{1,1}^{k, m}+b_{k m} \overline{z_{1,1}^{k, m}}+c_{k m}\left[\left(z_{1,1}^{k, m}\right)^{2}-{\overline{z_{1,1}^{k, m}}}^{2}\right]+d_{k m}, \\
& \rho\left(z_{0,1}^{k, m}\right)=a_{k m} z_{0,1}^{k, m}+b_{k m} \overline{z_{0,1}^{k, m}}+c_{k m}\left[\left(z_{0,1}^{k, m}\right)^{2}-{\overline{z_{0,1}^{k, m}}}^{2}\right]+d_{k m} .
\end{aligned}
$$

Solving (2), we obtain coefficients $a_{k m}, b_{k m}, c_{k m}$, and $d_{k m}$ as follows:

$$
\begin{aligned}
& a_{k, m}=Q_{k, m}^{-1} \cdot\left(r_{0,0}^{k, m} \cdot\left(z_{0,1}^{k, m}\right)^{2} \cdot \overline{z_{1,0}^{k, m}}-r_{0,0}^{k, m} \cdot\left(z_{1,0}^{k, m}\right)^{2} \cdot \overline{z_{0,1}^{k, m}}\right. \\
& -r_{0,1}^{k, m} \cdot\left(z_{0,0}^{k, m}\right)^{2} \cdot \overline{z_{1,0}^{k, m}}+r_{0,1}^{k, m} \cdot\left(z_{1,0}^{k, m}\right)^{2} \cdot \overline{z_{0,0}^{k, m}}+r_{1,0}^{k, m} \cdot\left(z_{0,0}^{k, m}\right)^{2} \cdot \overline{z_{0,1}^{k, m}}
\end{aligned}
$$




$$
\begin{aligned}
& -r_{1,0}^{k, m} \cdot\left(z_{0,1}^{k, m}\right)^{2} \cdot \overline{z_{0,0}^{k, m}}-r_{0,0}^{k, m} \cdot\left(z_{0,1}^{k, m}\right)^{2} \cdot \overline{z_{1,1}^{k, m}}+r_{0,0}^{k, m} \cdot\left(z_{1,1}^{k, m}\right)^{2} \cdot \overline{z_{0,1}^{k, m}} \\
& +r_{0,1}^{k, m} \cdot\left(z_{0,0}^{k, m}\right)^{2} \cdot \overline{z_{1,1}^{k, m}}-r_{0,1}^{k, m} \cdot\left(z_{1,1}^{k, m}\right)^{2} \cdot \overline{z_{0,0}^{k, m}}-r_{1,1}^{k, m} \cdot\left(z_{0,0}^{k, m}\right)^{2} \cdot \overline{z_{0,1}^{k, m}} \\
& +r_{1,1}^{k, m} \cdot\left(z_{0,1}^{k, m}\right)^{2} \cdot \overline{z_{0,0}^{k, m}}+r_{1,1}^{k, m} \cdot\left(z_{0,1}^{k, m}\right)^{2} \cdot \overline{z_{0,0}^{k, m}}-r_{0,0}^{k, m} \cdot\left(z_{1,0}^{k, m}\right)^{2} \cdot \overline{z_{1,1}^{k, m}} \\
& -r_{0,0}^{k, m} \cdot\left(z_{1,1}^{k, m}\right)^{2} \cdot \overline{z_{1,1}^{k, m}}+r_{1,0}^{k, m} \cdot\left(z_{1,1}^{k, m}\right)^{2} \cdot \overline{z_{0,0}^{k, m}}+r_{1,1}^{k, m} \cdot\left(z_{0,0}^{k, m}\right)^{2} \cdot \overline{z_{1,0}^{k, m}} \\
& -r_{1,1}^{k, m} \cdot\left(z_{1,0}^{k, m}\right)^{2} \cdot \overline{z_{1,1}^{k, m}}-r_{0,1}^{k, m} \cdot\left(z_{1,0}^{k, m}\right)^{2} \cdot \overline{z_{1,1}^{k, m}}+r_{0,1}^{k, m} \cdot\left(z_{1,1}^{k, m}\right)^{2} \cdot \overline{z_{1,0}^{k, m}} \\
& +r_{1,0}^{k, m} \cdot\left(z_{0,1}^{k, m}\right)^{2} \cdot \overline{z_{1,1}^{k, m}}-r_{1,0}^{k, m} \cdot\left(z_{1,1}^{k, m}\right)^{2} \cdot \overline{z_{0,1}^{k, m}}-r_{1,1}^{k, m} \cdot\left(z_{0,1}^{k, m}\right)^{2} \cdot \overline{z_{1,0}^{k, m}} \\
& +r_{1,1}^{k, m} \cdot\left(z_{1,0}^{k, m}\right)^{2} \cdot \overline{z_{0,1}^{k, m}}+r_{0,0}^{k, m} \cdot \overline{z_{0,1}^{k, m}} \cdot\left(\overline{z_{1,0}^{k, m}}\right)^{2}-r_{0,0}^{k, m} \cdot\left(\overline{z_{0,1}^{k, m}}\right)^{2} \cdot \overline{z_{1,0}^{k, m}} \\
& -r_{0,1}^{k, m} \cdot \overline{z_{0,0}^{k, m}} \cdot\left(\overline{z_{1,0}^{k, m}}\right)^{2}+r_{0,1}^{k, m} \cdot\left(\overline{z_{0,0}^{k, m}}\right)^{2} \cdot \overline{z_{1,0}^{k, m}}+r_{1,0}^{k, m} \cdot \overline{z_{0,0}^{k, m}} \cdot\left(\overline{z_{0,1}^{k, m}}\right)^{2} \\
& -r_{1,0}^{k, m} \cdot\left(\overline{z_{0,0}^{k, m}}\right)^{2} \cdot\left(\overline{z_{0,1}^{k, m}}\right)^{2}-r_{0,0}^{k, m} \cdot \overline{z_{0,1}^{k, m}}\left(\overline{z_{1,1}^{k, m}}\right)^{2}+r_{0,0}^{k, m} \cdot\left(\overline{z_{0,1}^{k, m}}\right)^{2} \overline{z_{1,1}^{k, m}} \\
& +r_{0,1}^{k, m} \cdot \overline{z_{0,0}^{k, m}} \cdot\left(\overline{z_{1,1}^{k, m}}\right)^{2}-r_{0,1}^{k, m} \cdot\left(\overline{z_{0,0}^{k, m}}\right)^{2} \cdot \overline{z_{1,1}^{k, m}}-r_{1,1}^{k, m} \cdot \overline{z_{0,0}^{k, m}} \cdot\left(\overline{z_{0,1}^{k, m}}\right)^{2} \\
& +r_{1,1}^{k, m} \cdot\left(\overline{z_{0,0}^{k, m}}\right)^{2} \cdot \overline{z_{0,1}^{k, m}}+r_{0,0}^{k, m} \cdot \overline{z_{1,0}^{k, m}} \cdot\left(\overline{z_{1,1}^{k, m}}\right)^{2}-r_{0,0}^{k, m} \cdot\left(\overline{z_{1,0}^{k, m}}\right)^{2} \cdot \overline{z_{1,1}^{k, m}} \\
& -r_{1,0}^{k, m} \cdot \overline{z_{0,0}^{k, m}} \cdot\left(\overline{z_{1,1}^{k, m}}\right)^{2}+r_{1,0}^{k, m} \cdot\left(\overline{z_{0,0}^{k, m}}\right)^{2} \cdot \overline{z_{1,1}^{k, m}}+r_{1,1}^{k, m} \cdot \overline{z_{0,0}^{k, m}} \cdot\left(\overline{z_{1,0}^{k, m}}\right)^{2} \\
& -r_{1,1}^{k, m} \cdot\left(\overline{z_{0,0}^{k, m}}\right)^{2} \cdot \overline{z_{1,0}^{k, m}}-r_{0,1}^{k, m} \cdot \overline{z_{1,0}^{k, m}} \cdot\left(\overline{z_{1,1}^{k, m}}\right)^{2}+r_{0,1}^{k, m} \cdot\left(\overline{z_{1,0}^{k, m}}\right)^{2} \cdot \overline{z_{1,1}^{k, m}} \\
& +r_{1,0}^{k, m} \cdot \overline{z_{0,1}^{k, m}} \cdot\left(\overline{z_{1,1}^{k, m}}\right)^{2}-r_{1,0}^{k, m} \cdot\left(\overline{z_{0,1}^{k, m}}\right)^{2} \cdot \overline{z_{1,1}^{k, m}}-r_{1,1}^{k, m} \cdot \overline{z_{0,1}^{k, m}} \cdot\left(\overline{z_{1,0}^{k, m}}\right)^{2} \\
& \left.+r_{1,1}^{k, m} \cdot\left(\overline{z_{0,1}^{k, m}}\right)^{2} \cdot \overline{z_{1,0}^{k, m}}\right)
\end{aligned}
$$

$b_{k, m}=Q_{k, m}^{-1} \cdot\left(r_{0,0}^{k, m} \cdot z_{0,1}^{k, m} \cdot\left(z_{1,0}^{k, m}\right)^{2}-r_{0,0}^{k, m} \cdot\left(z_{0,1}^{k, m}\right)^{2} \cdot z_{1,0}^{k, m}\right.$

$-r_{0,1}^{k, m} \cdot z_{0,0}^{k, m} \cdot\left(z_{1,0}^{k, m}\right)^{2}+r_{0,1}^{k, m} \cdot\left(z_{0,0}^{k, m}\right)^{2} \cdot z_{1,0}^{k, m}+r_{1,0}^{k, m} \cdot z_{0,0}^{k, m} \cdot\left(z_{0,1}^{k, m}\right)^{2}$

$-r_{1,0}^{k, m} \cdot\left(z_{0,0}^{k, m}\right)^{2} \cdot z_{0,1}^{k, m}-r_{0,0}^{k, m} \cdot z_{0,1}^{k, m} \cdot\left(z_{1,1}^{k, m}\right)^{2}+r_{0,0}^{k, m} \cdot\left(z_{0,1}^{k, m}\right)^{2} \cdot z_{1,1}^{k, m}$

$+r_{0,1}^{k, m} \cdot z_{0,0}^{k, m} \cdot\left(z_{1,1}^{k, m}\right)^{2}-r_{0,1}^{k, m} \cdot\left(z_{0,0}^{k, m}\right)^{2} \cdot z_{1,1}^{k, m}-r_{1,1}^{k, m} \cdot z_{0,0}^{k, m} \cdot\left(z_{0,1}^{k, m}\right)^{2}$ 


$$
\begin{aligned}
& +r_{1,1}^{k, m} \cdot\left(z_{0,0}^{k, m}\right)^{2} \cdot z_{0,1}^{k, m}+r_{0,0}^{k, m} \cdot z_{1,0}^{k, m} \cdot\left(z_{1,1}^{k, m}\right)^{2}-r_{0,0}^{k, m} \cdot\left(z_{1,0}^{k, m}\right)^{2} \cdot z_{1,1}^{k, m} \\
& -r_{1,0}^{k, m} \cdot z_{0,0}^{k, m} \cdot\left(z_{1,1}^{k, m}\right)^{2}+r_{1,0}^{k, m} \cdot\left(z_{0,0}^{k, m}\right)^{2} \cdot z_{1,1}^{k, m}+r_{1,1}^{k, m} \cdot z_{0,0}^{k, m} \cdot\left(z_{1,0}^{k, m}\right)^{2} \\
& -r_{1,1}^{k, m} \cdot\left(z_{0,0}^{k, m}\right)^{2} \cdot z_{1,0}^{k, m}-r_{0,1}^{k, m} \cdot z_{1,0}^{k, m} \cdot\left(z_{1,1}^{k, m}\right)^{2}+r_{0,1}^{k, m} \cdot\left(z_{1,0}^{k, m}\right)^{2} \cdot z_{1,1}^{k, m} \\
& +r_{1,0}^{k, m} \cdot z_{0,1}^{k, m} \cdot\left(z_{1,1}^{k, m}\right)^{2}-r_{1,0}^{k, m} \cdot\left(z_{0,1}^{k, m}\right)^{2} \cdot z_{1,1}^{k, m}-r_{1,1}^{k, m} \cdot z_{0,1}^{k, m} \cdot\left(z_{1,0}^{k, m}\right)^{2} \\
& +r_{1,1}^{k, m} \cdot\left(z_{0,1}^{k, m}\right)^{2} \cdot z_{1,0}^{k, m}-r_{0,0}^{k, m} \cdot z_{0,1}^{k, m} \cdot\left(\overline{z_{1,0}^{k, m}}\right)^{2}+r_{0,0}^{k, m} \cdot z_{1,0}^{k, m} \cdot\left(\overline{z_{0,1}^{k, m}}\right)^{2} \\
& +r_{0,1}^{k, m} \cdot z_{0,0}^{k, m} \cdot\left(\overline{z_{1,0}^{k, m}}\right)^{2}-r_{0,1}^{k, m} \cdot z_{1,0}^{k, m} \cdot\left(\overline{z_{0,0}^{k, m}}\right)^{2}-r_{1,0}^{k, m} \cdot z_{0,0}^{k, m}\left(\overline{z_{0,1}^{k, m}}\right)^{2} \\
& +r_{1,0}^{k, m} \cdot z_{0,1}^{k, m} \cdot\left(\overline{z_{0,0}^{k, m}}\right)^{2}+r_{0,0}^{k, m} \cdot z_{0,1}^{k, m} \cdot\left(\overline{z_{1,1}^{k, m}}\right)^{2}-r_{0,0}^{k, m} \cdot z_{1,1}^{k, m} \cdot\left(\overline{z_{0,1}^{k, m}}\right)^{2} \\
& -r_{0,1}^{k, m} \cdot z_{0,0}^{k, m} \cdot\left(\overline{z_{1,1}^{k, m}}\right)^{2}+r_{0,1}^{k, m} \cdot z_{1,1}^{k, m} \cdot\left(\overline{z_{0,0}^{k, m}}\right)^{2}+r_{1,1}^{k, m} \cdot z_{0,0}^{k, m} \cdot\left(\overline{z_{0,1}^{k, m}}\right)^{2} \\
& -r_{1,1}^{k, m} \cdot z_{0,1}^{k, m} \cdot\left(\overline{z_{0,0}^{k, m}}\right)^{2}-r_{0,0}^{k, m} \cdot z_{1,0}^{k, m} \cdot\left(\overline{z_{1,1}^{k, m}}\right)^{2}+r_{0,0}^{k, m} \cdot z_{1,1}^{k, m} \cdot\left(\overline{z_{1,0}^{k, m}}\right)^{2} \\
& +r_{1,0}^{k, m} \cdot z_{0,0}^{k, m} \cdot\left(\overline{z_{1,1}^{k, m}}\right)^{2}-r_{1,0}^{k, m} \cdot z_{1,1}^{k, m} \cdot\left(\overline{z_{0,0}^{k, m}}\right)^{2}-r_{1,1}^{k, m} \cdot z_{0,0}^{k, m} \cdot\left(\overline{z_{1,0}^{k, m}}\right)^{2} \\
& +r_{1,1}^{k, m} \cdot z_{1,0}^{k, m} \cdot\left(\overline{z_{0,0}^{k, m}}\right)^{2}+r_{0,1}^{k, m} \cdot z_{1,0}^{k, m} \cdot\left(\overline{z_{1,1}^{k, m}}\right)^{2}-r_{0,1}^{k, m} \cdot z_{1,1}^{k, m} \cdot\left(\overline{z_{1,0}^{k, m}}\right)^{2} \\
& -r_{1,0}^{k, m} \cdot z_{0,1}^{k, m} \cdot\left(\overline{z_{1,1}^{k, m}}\right)^{2}+r_{1,0}^{k, m} \cdot z_{1,1}^{k, m} \cdot\left(\overline{z_{0,1}^{k, m}}\right)^{2}+r_{1,1}^{k, m} \cdot z_{0,1}^{k, m} \cdot\left(\overline{z_{1,0}^{k, m}}\right)^{2} \\
& \left.-r_{1,1}^{k, m} \cdot z_{1,0}^{k, m} \cdot\left(\overline{z_{0,1}^{k, m}}\right)^{2}\right),
\end{aligned}
$$

$$
c_{k, m}=-Q_{k, m}^{-1} \cdot\left(r_{0,0}^{k, m} \cdot z_{0,1}^{k, m} \cdot \overline{z_{1,0}^{k, m}}-r_{0,0}^{k, m} \cdot z_{1,0}^{k, m} \cdot \overline{z_{0,1}^{k, m}}-r_{0,1}^{k, m} \cdot z_{0,0}^{k, m} \cdot \overline{z_{1,0}^{k, m}}\right.
$$$$
+r_{0,1}^{k, m} \cdot z_{1,0}^{k, m} \cdot \overline{z_{0,0}^{k, m}}+r_{1,0}^{k, m} \cdot z_{0,0}^{k, m} \cdot \overline{z_{0,1}^{k, m}}-r_{0,1}^{k, m} \cdot z_{0,1}^{k, m} \cdot \overline{z_{0,0}^{k, m}}-r_{0,0}^{k, m} \cdot z_{0,1}^{k, m} \cdot \overline{z_{1,1}^{k, m}}
$$$$
+r_{0,0}^{k, m} \cdot z_{1,1}^{k, m} \cdot \overline{z_{0,1}^{k, m}}+r_{0,1}^{k, m} \cdot z_{0,0}^{k, m} \cdot \overline{z_{1,1}^{k, m}}-r_{0,1}^{k, m} \cdot z_{1,1}^{k, m} \cdot \overline{z_{0,0}^{k, m}}-r_{1,1}^{k, m} \cdot z_{0,0}^{k, m} \cdot \overline{z_{0,1}^{k, m}}
$$$$
+r_{1,1}^{k, m} \cdot z_{0,1}^{k, m} \cdot \overline{z_{0,0}^{k, m}}+r_{0,0}^{k, m} \cdot z_{1,0}^{k, m} \cdot \overline{z_{1,1}^{k, m}}-r_{0,0}^{k, m} \cdot z_{1,1}^{k, m} \cdot \overline{z_{1,0}^{k, m}}-r_{1,0}^{k, m} \cdot z_{0,0}^{k, m} \cdot \overline{z_{0,1}^{k, m}}
$$$$
+r_{1,0}^{k, m} \cdot z_{1,1}^{k, m} \cdot \overline{z_{0,0}^{k, m}}+r_{1,1}^{k, m} \cdot z_{0,0}^{k, m} \cdot \overline{z_{1,0}^{k, m}}-r_{1,1}^{k, m} \cdot z_{1,0}^{k, m} \cdot \overline{z_{0,0}^{k, m}}-r_{0,1}^{k, m} \cdot z_{1,0}^{k, m} \cdot \overline{z_{1,0}^{k, m}}
$$$$
+r_{0,1}^{k, m} \cdot z_{1,1}^{k, m} \cdot \overline{z_{1,0}^{k, m}}+r_{1,0}^{k, m} \cdot z_{0,1}^{k, m} \cdot \overline{z_{1,1}^{k, m}}-r_{1,0}^{k, m} \cdot z_{1,1}^{k, m} \cdot \overline{z_{0,1}^{k, m}}-r_{1,1}^{k, m} \cdot z_{0,1}^{k, m} \cdot \overline{z_{1,0}^{k, m}}
$$$$
\left.+r_{1,1}^{k, m} \cdot z_{1,0}^{k, m} \cdot \overline{z_{0,1}^{k, m}}\right)
$$ 


$$
\begin{aligned}
& d_{k, m}=Q_{k, m}^{-1} \cdot\left(r_{0,0}^{k, m} \cdot z_{0,1}^{k, m} \cdot\left(z_{1,0}^{k, m}\right)^{2} \cdot \overline{z_{1,1}^{k, m}}-r_{0,0}^{k, m} \cdot z_{0,1}^{k, m} \cdot\left(z_{1,1}^{k, m}\right)^{2} \cdot \overline{z_{1,0}^{k, m}}\right. \\
& +r_{0,0}^{k, m} \cdot z_{1,0}^{k, m} \cdot\left(z_{1,1}^{k, m}\right)^{2} \cdot \overline{z_{0,1}^{k, m}}-r_{0,0}^{k, m} \cdot\left(z_{0,1}^{k, m}\right)^{2} \cdot z_{1,0}^{k, m} \cdot \overline{z_{1,1}^{k, m}} \\
& +r_{0,0}^{k, m} \cdot\left(z_{0,1}^{k, m}\right)^{2} \cdot z_{1,1}^{k, m} \cdot \overline{z_{1,0}^{k, m}}-r_{0,0}^{k, m} \cdot\left(z_{1,0}^{k, m}\right)^{2} \cdot z_{1,1}^{k, m} \cdot \overline{z_{0,1}^{k, m}} \\
& -r_{0,1}^{k, m} \cdot z_{0,0}^{k, m} \cdot\left(z_{1,0}^{k, m}\right)^{2} \cdot \overline{z_{1,1}^{k, m}}+r_{0,1}^{k, m} \cdot z_{0,0}^{k, m} \cdot\left(z_{1,1}^{k, m}\right)^{2} \cdot \overline{z_{1,0}^{k, m}} \\
& -r_{0,1}^{k, m} \cdot z_{1,0}^{k, m} \cdot\left(z_{1,1}^{k, m}\right)^{2} \cdot \overline{z_{0,0}^{k, m}}+r_{0,1}^{k, m} \cdot\left(z_{0,0}^{k, m}\right)^{2} \cdot z_{1,0}^{k, m} \cdot \overline{z_{1,1}^{k, m}} \\
& -r_{0,1}^{k, m} \cdot\left(z_{0,0}^{k, m}\right)^{2} \cdot z_{1,1}^{k, m} \cdot \overline{z_{1,0}^{k, m}}+r_{0,1}^{k, m} \cdot\left(z_{1,0}^{k, m}\right)^{2} \cdot z_{1,1}^{k, m} \cdot \overline{z_{0,0}^{k, m}} \\
& +r_{1,0}^{k, m} \cdot z_{0,0}^{k, m} \cdot\left(z_{0,1}^{k, m}\right)^{2} \cdot \overline{z_{1,1}^{k, m}}-r_{1,0}^{k, m} \cdot z_{0,0}^{k, m} \cdot\left(z_{1,1}^{k, m}\right)^{2} \cdot \overline{z_{0,1}^{k, m}} \\
& +r_{1,0}^{k, m} \cdot z_{0,1}^{k, m} \cdot\left(z_{1,1}^{k, m}\right)^{2} \cdot \overline{z_{0,0}^{k, m}}-r_{1,0}^{k, m} \cdot\left(z_{0,0}^{k, m}\right)^{2} \cdot z_{0,1}^{k, m} \cdot \overline{z_{1,1}^{k, m}} \\
& +r_{1,0}^{k, m} \cdot\left(z_{0,0}^{k, m}\right)^{2} \cdot z_{1,1}^{k, m} \cdot \overline{z_{0,1}^{k, m}}-r_{1,0}^{k, m} \cdot\left(z_{0,1}^{k, m}\right)^{2} \cdot z_{1,1}^{k, m} \cdot \overline{z_{0,0}^{k, m}} \\
& -r_{1,1}^{k, m} \cdot z_{0,0}^{k, m} \cdot\left(z_{0,1}^{k, m}\right)^{2} \cdot \overline{z_{1,0}^{k, m}}+r_{1,1}^{k, m} \cdot z_{0,0}^{k, m} \cdot\left(z_{1,0}^{k, m}\right)^{2} \cdot \overline{z_{0,1}^{k, m}} \\
& -r_{1,1}^{k, m} \cdot z_{0,1}^{k, m} \cdot\left(z_{1,0}^{k, m}\right)^{2} \cdot \overline{z_{0,0}^{k, m}}+r_{1,1}^{k, m} \cdot\left(z_{0,0}^{k, m}\right)^{2} \cdot z_{0,1}^{k, m} \cdot \overline{z_{1,0}^{k, m}} \\
& -r_{1,1}^{k, m} \cdot\left(z_{0,0}^{k, m}\right)^{2} \cdot z_{1,0}^{k, m} \cdot \overline{z_{0,1}^{k, m}}+r_{1,1}^{k, m} \cdot\left(z_{0,1}^{k, m}\right)^{2} \cdot z_{1,0}^{k, m} \cdot \overline{z_{0,0}^{k, m}} \\
& +r_{0,0}^{k, m} \cdot z_{0,1}^{k, m} \cdot \overline{z_{1,0}^{k, m}} \cdot\left(\overline{z_{1,1}^{k, m}}\right)^{2}-r_{0,0}^{k, m} \cdot z_{0,1}^{k, m} \cdot\left(\overline{z_{1,0}^{k, m}}\right)^{2} \cdot \overline{z_{1,1}^{k, m}} \\
& -r_{0,0}^{k, m} \cdot z_{1,0}^{k, m} \cdot \overline{z_{0,1}^{k, m}} \cdot\left(\overline{z_{1,1}^{k, m}}\right)^{2}+r_{0,0}^{k, m} \cdot z_{1,0}^{k, m} \cdot\left(\overline{z_{0,1}^{k, m}}\right)^{2} \cdot \overline{z_{1,1}^{k, m}} \\
& +r_{0,0}^{k, m} \cdot z_{1,1}^{k, m} \cdot \overline{z_{0,1}^{k, m}} \cdot\left(\overline{z_{1,0}^{k, m}}\right)^{2}-r_{0,0}^{k, m} \cdot z_{1,1}^{k, m} \cdot\left(\overline{z_{0,1}^{k, m}}\right)^{2} \cdot \overline{z_{1,0}^{k, m}} \\
& -r_{0,1}^{k, m} \cdot z_{0,0}^{k, m} \cdot \overline{z_{1,0}^{k, m}} \cdot\left(\overline{z_{1,1}^{k, m}}\right)^{2}+r_{0,1}^{k, m} \cdot z_{0,0}^{k, m} \cdot\left(\overline{z_{1,0}^{k, m}}\right)^{2} \cdot \overline{z_{1,1}^{k, m}} \\
& +r_{0,1}^{k, m} \cdot z_{1,0}^{k, m} \cdot \overline{z_{0,0}^{k, m}} \cdot\left(\overline{z_{1,1}^{k, m}}\right)^{2}-r_{0,1}^{k, m} \cdot z_{1,0}^{k, m} \cdot\left(\overline{z_{0,0}^{k, m}}\right)^{2} \cdot \overline{z_{1,1}^{k, m}} \\
& -r_{0,1}^{k, m} \cdot z_{1,1}^{k, m} \cdot \overline{z_{0,0}^{k, m}} \cdot\left(\overline{z_{1,0}^{k, m}}\right)^{2}+r_{0,1}^{k, m} \cdot z_{1,1}^{k, m} \cdot\left(\overline{z_{0,0}^{k, m}}\right)^{2} \cdot \overline{z_{1,0}^{k, m}} \\
& +r_{1,0}^{k, m} \cdot z_{0,0}^{k, m} \cdot \overline{z_{0,1}^{k, m}} \cdot\left(\overline{z_{1,1}^{k, m}}\right)^{2}-r_{1,0}^{k, m} \cdot z_{0,0}^{k, m} \cdot\left(\overline{z_{0,1}^{k, m}}\right)^{2} \cdot \overline{z_{1,1}^{k, m}} \\
& -r_{1,0}^{k, m} \cdot z_{0,1}^{k, m} \cdot \overline{z_{0,0}^{k, m}} \cdot\left(\overline{z_{1,1}^{k, m}}\right)^{2}+r_{1,0}^{k, m} \cdot z_{0,1}^{k, m} \cdot\left(\overline{z_{0,0}^{k, m}}\right)^{2} \cdot \overline{z_{1,1}^{k, m}} \\
& +r_{1,0}^{k, m} \cdot z_{1,1}^{k, m} \cdot \overline{z_{0,0}^{k, m}} \cdot\left(\overline{z_{0,1}^{k, m}}\right)^{2}-r_{1,0}^{k, m} \cdot z_{1,1}^{k, m} \cdot\left(\overline{z_{0,0}^{k, m}}\right)^{2} \cdot \overline{z_{0,1}^{k, m}} \\
& -r_{1,1}^{k, m} \cdot z_{0,0}^{k, m} \cdot \overline{z_{0,1}^{k, m}} \cdot\left(\overline{z_{1,0}^{k, m}}\right)^{2}+r_{1,1}^{k, m} \cdot z_{0,0}^{k, m} \cdot\left(\overline{z_{0,1}^{k, m}}\right)^{2} \cdot \overline{z_{1,0}^{k, m}} \\
& +r_{1,1}^{k, m} \cdot z_{0,1}^{k, m} \cdot \overline{z_{0,0}^{k, m}} \cdot\left(\overline{z_{0,1}^{k, m}}\right)^{2}-r_{1,1}^{k, m} \cdot z_{0,1}^{k, m} \cdot\left(\overline{z_{0,0}^{k, m}}\right)^{2} \cdot \overline{z_{1,0}^{k, m}} \\
& \left.-r_{1,1}^{k, m} \cdot z_{1,0}^{k, m} \cdot \overline{z_{0,0}^{k, m}} \cdot\left(\overline{z_{0,1}^{k, m}}\right)^{2}+r_{1,1}^{k, m} \cdot z_{1,0}^{k, m} \cdot\left(\overline{z_{0,0}^{k, m}}\right)^{2} \cdot \overline{z_{0,1}^{k, m}}\right) \text {, }
\end{aligned}
$$


where

$$
\begin{aligned}
& Q_{k, m}=z_{0,0}^{k, m} \cdot\left(z_{0,1}^{k, m}\right)^{2} \cdot \overline{z_{1,0}^{k, m}}-z_{0,0}^{k, m} \cdot\left(z_{1,0}^{k, m}\right)^{2} \cdot \overline{z_{0,1}^{k, m}}+z_{0,1}^{k, m} \cdot\left(z_{1,0}^{k, m}\right)^{2} \cdot z_{0,0}^{k, m} \\
& -\left(z_{0,0}^{k, m}\right)^{2} \cdot z_{0,1}^{k, m} \cdot \overline{z_{1,0}^{k, m}}+\left(z_{0,0}^{k, m}\right)^{2} \cdot z_{1,0}^{k, m} \overline{z_{0,1}^{k, m}}-\left(z_{0,1}^{k, m}\right)^{2} \cdot z_{1,0}^{k, m} \cdot \overline{z_{0,0}^{k, m}} \\
& -z_{0,0}^{k, m} \cdot\left(z_{0,1}^{k, m}\right)^{2} \cdot \overline{z_{1,1}^{k, m}}+z_{0,0}^{k, m}\left(z_{1,1}^{k, m}\right)^{2} \cdot \overline{z_{0,1}^{k, m}}-z_{0,1}^{k, m} \cdot\left(z_{1,1}^{k, m}\right)^{2} \cdot \overline{z_{0,0}^{k, m}} \\
& +\left(z_{0,0}^{k, m}\right)^{2} \cdot z_{0,1}^{k, m} \cdot \overline{z_{1,1}^{k, m}}-\left(z_{0,0}^{k, m}\right)^{2} \cdot z_{1,1}^{k, m} \cdot \overline{z_{0,1}^{k, m}}+\left(z_{0,1}^{k, m}\right)^{2} \cdot z_{1,1}^{k, m} \overline{z_{0,0}^{k, m}} \\
& +z_{0,0}^{k, m} \cdot\left(z_{1,0}^{k, m}\right)^{2} \cdot \overline{z_{1,1}^{k, m}}-z_{0,0}^{k, m} \cdot\left(z_{1,1}^{k, m}\right)^{2} \cdot \overline{z_{1,0}^{k, m}}+z_{1,0}^{k, m} \cdot\left(z_{1,1}^{k, m}\right)^{2} \cdot \overline{z_{0,0}^{k, m}} \\
& -\left(z_{0,0}^{k, m}\right)^{2} \cdot z_{1,0}^{k, m} \cdot \overline{z_{1,1}^{k, m}}+\left(z_{0,0}^{k, m}\right)^{2} \cdot z_{1,1}^{k, m} \overline{z_{1,0}^{k, m}}-\left(z_{1,0}^{k, m}\right)^{2} \cdot z_{1,1}^{k, m} \overline{z_{0,0}^{k, m}} \\
& -z_{0,1}^{k, m} \cdot\left(z_{1,0}^{k, m}\right)^{2} \cdot \overline{z_{1,1}^{k, m}}+z_{0,1}^{k, m} \cdot\left(z_{1,1}^{k, m}\right)^{2} \cdot \overline{z_{1,0}^{k, m}}-z_{1,0}^{k, m} \cdot\left(z_{1,1}^{k, m}\right)^{2} \cdot \overline{z_{0,1}^{k, m}} \\
& +\left(z_{0,1}^{k, m}\right)^{2} \cdot z_{1,0}^{k, m} \cdot \overline{z_{1,1}^{k, m}}-\left(z_{0,1}^{k, m}\right)^{2} \cdot z_{1,1}^{k, m} \cdot \overline{z_{1,0}^{k, m}} \\
& +\left(z_{1,0}^{k, m}\right)^{2} \cdot z_{1,1}^{k, m} \cdot \overline{z_{0,1}^{k, m}}+z_{0,0}^{k, m} \cdot \overline{z_{0,1}^{k, m}} \cdot\left(\overline{z_{1,0}^{k, m}}\right)^{2}-z_{0,0}^{k, m} \cdot\left(\overline{z_{0,1}^{k, m}}\right)^{2} \cdot \overline{z_{1,0}^{k, m}} \\
& -z_{0,1}^{k, m} \cdot \overline{z_{0,0}^{k, m}} \cdot\left(\overline{z_{1,0}^{k, m}}\right)^{2}+z_{0,1}^{k, m} \cdot\left(\overline{z_{0,0}^{k, m}}\right)^{2} \cdot \overline{z_{1,0}^{k, m}}+z_{1,0}^{k, m} \cdot \overline{z_{0,0}^{k, m}} \cdot\left(\overline{z_{0,1}^{k, m}}\right)^{2} \\
& -z_{1,0}^{k, m} \cdot\left(\overline{z_{0,0}^{k, m}}\right)^{2} \cdot \overline{z_{0,1}^{k, m}}-z_{0,0}^{k, m} \cdot \overline{z_{0,1}^{k, m}} \cdot\left(\overline{z_{1,1}^{k, m}}\right)^{2}+z_{0,0}^{k, m} \cdot\left(\overline{z_{0,1}^{k, m}}\right)^{2} \cdot \overline{z_{1,1}^{k, m}} \\
& +z_{0,1}^{k, m} \cdot \overline{z_{0,0}^{k, m}} \cdot\left(\overline{z_{1,1}^{k, m}}\right)^{2}-z_{0,1}^{k, m} \cdot\left(\overline{z_{0,0}^{k, m}}\right)^{2} \cdot \overline{z_{1,1}^{k, m}}-z_{1,1}^{k, m} \cdot \overline{z_{0,0}^{k, m}} \cdot\left(\overline{z_{0,1}^{k, m}}\right)^{2} \\
& z_{1,1}^{k, m} \cdot\left(\overline{z_{0,0}^{k, m}}\right)^{2} \cdot \overline{z_{0,1}^{k, m}}+z_{0,0}^{k, m} \cdot \overline{z_{1,0}^{k, m}} \cdot\left(\overline{z_{1,1}^{k, m}}\right)^{2}-z_{0,0}^{k, m} \cdot\left(\overline{z_{1,0}^{k, m}}\right)^{2} \cdot \overline{z_{1,1}^{k, m}} \\
& -z_{1,0}^{k, m} \cdot \overline{z_{0,0}^{k, m}} \cdot\left(\overline{z_{1,1}^{k, m}}\right)^{2}+z_{1,0}^{k, m} \cdot\left(\overline{z_{0,0}^{k, m}}\right)^{2} \cdot \overline{z_{1,1}^{k, m}}+z_{1,1}^{k, m} \cdot \overline{z_{0,0}^{k, m}} \cdot\left(\overline{z_{1,0}^{k, m}}\right)^{2} \\
& -z_{1,1}^{k, m} \cdot\left(\overline{z_{0,0}^{k, m}}\right)^{2} \cdot \overline{z_{1,0}^{k, m}}-z_{0,1}^{k, m} \cdot \overline{z_{1,0}^{k, m}} \cdot\left(\overline{z_{1,1}^{k, m}}\right)^{2}+z_{0,1}^{k, m} \cdot\left(\overline{z_{1,0}^{k, m}}\right)^{2} \cdot \overline{z_{1,1}^{k, m}} \\
& +z_{1,0}^{k, m} \overline{z_{0,1}^{k, m}} \cdot\left(\overline{z_{1,1}^{k, m}}\right)^{2}-z_{1,0}^{k, m} \cdot\left(\overline{z_{0,1}^{k, m}}\right)^{2} \cdot \overline{z_{1,1}^{k, m}}-z_{1,1}^{k, m} \cdot \overline{z_{0,1}^{k, m}} \cdot\left(\overline{z_{1,0}^{k, m}}\right)^{2} \\
& \left.+z_{1,1}^{k, m} \cdot\left(\overline{z_{0,1}^{k, m}}\right)^{2} \cdot \overline{z_{1,0}^{k, m}}\right) \text {. }
\end{aligned}
$$

\section{Approximation of $T$}

In this section, we give quadrature formula for potential integral $T$. By using spline approximation of $\rho$ in the domain $D_{k, m}$, we have

$$
\begin{aligned}
& T(\widehat{\rho} \mid z)=-\frac{1}{\pi} \sum_{k=1}^{N-1} \sum_{m=0}^{M_{k}-1}\left(a_{k, m} \iint_{D_{k, m}} \frac{\zeta d \zeta}{\zeta-z}+b_{k, m} \iint \frac{\bar{\zeta} d \zeta}{D_{k, m}-z}\right. \\
& \left.+c_{k, m} \iint_{D_{k, m}} \frac{\left(\zeta^{2}-\bar{\zeta}^{2}\right) d \zeta}{\zeta-z}+d_{k, m} \iint \frac{d \zeta}{D_{k, m}}\right)
\end{aligned}
$$




$$
\begin{aligned}
& =\sum_{k=1}^{N-1} \sum_{m=0}^{M_{k}-1}\left(a_{k, m} T_{k, m}^{a}(z)+b_{k, m} T_{k, m}^{b}(z)\right. \\
& \left.+c_{k, m} T_{k, m}^{c}(z)+d_{k, m} T_{k, m}^{d}(z)\right)
\end{aligned}
$$

where

$$
\begin{gathered}
T_{k, m}^{a}(z)=-\frac{1}{\pi} \iint_{D_{k, m}} \frac{\zeta d \zeta}{\zeta-z}, T_{k, m}^{b}(z)=-\frac{1}{\pi} \iint_{D_{k, m}} \frac{\bar{\zeta} d \zeta}{\zeta-z} \\
T_{k, m}^{c}(z)=-\frac{1}{\pi} \iint_{D_{k, m}} \frac{\left(\zeta^{2}-\bar{\zeta}^{2}\right) d \zeta}{\zeta-z}, T_{k, m}^{d}(z)=-\frac{1}{\pi} \iint_{D_{k, m}} \frac{d \zeta}{\zeta-z} .
\end{gathered}
$$

Let us evaluate $T_{k, m}^{a}$. By using Pompeu formula (see [13, p. 26, formula (4.15)]), two-dimensional integral can be reduced to one dimensional integral on boundary $\Gamma_{k, m}$. From identity $\frac{\partial(\zeta \bar{\zeta})}{\partial \bar{\zeta}}=\zeta$ on $\Gamma_{k, m}^{1}$, it follows that

$$
T_{k, m}^{a}(z)=\int_{\Gamma_{k, m}} \frac{\zeta \bar{\zeta}}{\zeta-z} d \zeta=\sum_{j=1}^{4} \int_{\Gamma_{k, m}^{j}} \frac{\zeta \bar{\zeta}}{\zeta-z} d \zeta
$$

In sequel,

$$
\begin{aligned}
& T_{k, m}^{a, 1}(z)=\int_{\Gamma_{k, m}^{1}} \frac{\zeta \bar{\zeta}}{\zeta-z} d \zeta=e^{-2 i \theta_{k, m}} \int_{\Gamma_{k, m}^{1}} \frac{\zeta^{2}}{\zeta-z} d \zeta \\
& =e^{-2 i \theta_{k, m}} \int_{\Gamma_{k, m}^{1}} \frac{\zeta^{2}-z^{2}+z^{2}}{\zeta-z} d \zeta \\
& =e^{-2 i \theta_{k, m}}\left[\int_{\Gamma_{k, m}^{1}} \frac{\zeta^{2}-z^{2}}{\zeta-z} d \zeta+z^{2} \int_{\Gamma_{k, m}^{1}} \frac{d \zeta}{\zeta-z}\right] \\
& =e^{-2 i \theta_{k, m}}\left[\frac{\zeta^{2}}{2}+z \zeta+z^{2} \ln (\zeta-z)\right]_{z_{0,0}^{k, m}}^{k, m} \\
& =\frac{z_{0,0}^{k, m}}{z_{0,0}^{k, m}}\left[\frac{\left(z_{1,0}^{k, m}\right)^{2}}{2}-\frac{\left(z_{0,0}^{k, m}\right)^{2}}{2}+z z_{1,0}^{k, m}-z z_{0,0}^{k, m}+z^{2} \ln \left|\frac{z_{1,0}^{k, m}-z}{z_{0,0}^{k, m}-z}\right|\right] .
\end{aligned}
$$

We have $\zeta \bar{\zeta}=r_{k+1} e^{i \theta} r_{k+1} e^{-i \theta}=r_{k+1}^{2}$ on $\Gamma_{k, m}^{2}$. Thus,

$$
\begin{gathered}
T_{k, m}^{a, 2}(z)=\int_{\Gamma_{k, m}^{2}} \frac{\zeta \bar{\zeta}}{\zeta-z} d \zeta=r_{k+1}^{2} \int_{\Gamma_{k, m}^{2}} \frac{d \zeta}{\zeta-z} \\
=\left.r_{k+1}^{2} \ln (\zeta-z)\right|_{z_{1,0}^{k, m}} ^{z_{1, m}^{k, m}}=z_{1,0}^{k, m} \overline{z_{1,0}^{k, m}} \ln \frac{z_{1,1}^{k, m}-z}{z_{1,0}^{k, m}-z} .
\end{gathered}
$$


For any $\zeta \in \Gamma_{k, m}^{3}$, the relation $\zeta \bar{\zeta}=r e^{i \theta_{k, m}} r e^{-i \theta_{k, m}}=r^{2}=\zeta^{2} e^{-2 i \theta_{k, m}}$ is true. So, we get

$$
\begin{aligned}
& T_{k, m}^{a, 3}(z)=\int_{\Gamma_{k, m}^{3}} \frac{\zeta \bar{\zeta}}{\zeta-z} d \zeta=e^{-2 i \theta_{k, m}} \int_{\Gamma_{k, m}^{3}} \frac{\zeta^{2}-z^{2}+z^{2}}{\zeta-z} d \zeta \\
& =e^{-2 i \theta_{k, m}}\left(\int_{\Gamma_{k, m}^{3}}(\zeta+z) d \zeta+\int_{\Gamma_{k, m}^{3}} \frac{z^{2}}{\zeta-z} d \zeta\right) \\
& =\frac{\overline{z_{1,1}^{k, m}}}{z_{1,1}^{k, m}}\left[\frac{\left(z_{0,1}^{k, m}\right)^{2}}{2}-\frac{\left(z_{1,1}^{k, m}\right)^{2}}{2}+z z_{0,1}^{k, m}-z z_{1,1}^{k, m}+z^{2} \ln \left|\frac{z_{0,1}^{k, m}-z}{z_{1,1}^{k, m}-z}\right|\right] .
\end{aligned}
$$

Since $\zeta \bar{\zeta}=r_{k} e^{i \theta} r_{k} e^{-i \theta}=r_{k}^{2}=z_{k, m} \overline{z_{k, m}}$ on $\Gamma_{k, m}^{4}$, we get

$$
\begin{gathered}
T_{k, m}^{a, 4}(z)=\int_{\Gamma_{k, m}^{4}} \frac{\zeta \bar{\zeta}}{\zeta-z} d \zeta=r_{k}^{2} \int_{\Gamma_{k, m}^{4}} \frac{d \zeta}{\zeta-z} \\
=r_{k}^{2}(\ln (\zeta-z))_{z_{0,1}^{k, m}}^{z_{0, m}^{k, m}}=z_{0,0}^{k, m} \overline{z_{0,0}^{k, m}} \ln \frac{z_{0,0}^{k, m}-z}{z_{0,1}^{k, m}-z} .
\end{gathered}
$$

By virtue (10)- (14), we have formula

$$
\begin{array}{r}
T_{k, m}^{a}(z)=\frac{\overline{z_{0,0}^{k, m}}}{z_{0,0}^{k, m}}\left[\frac{\left(z_{1,0}^{k, m}\right)^{2}}{2}-\frac{\left(z_{0,0}^{k, m}\right)^{2}}{2}+z z_{1,0}^{k, m}-z z_{0,0}^{k, m}+z^{2} \ln \left|\frac{z_{1,0}^{k, m}-z}{z_{0,0}^{k, m}-z}\right|\right] \\
\quad+z_{1,0}^{k, m} \overline{z_{1,0}^{k, m}} \ln \frac{z_{1,1}^{k, m}-z}{z_{1,0}^{k, m}-z}+z_{0,0}^{k, m} \overline{z_{0,0}^{k, m}} \ln \frac{z_{0,0}^{k, m}-z}{z_{0,1}^{k, m}-z} \\
+\frac{z_{1,1}^{k, m}}{z_{1,1}^{k, m}}\left[\frac{z_{0,1}^{k, m^{2}}}{2}-\frac{\left(z_{1,1}^{k, m}\right)^{2}}{2}+z z_{0,1}^{k, m}-z z_{1,1}^{k, m}+z^{2} \ln \left|\frac{z_{0,1}^{k, m}-z}{z_{1,1}^{k, m}-z}\right|\right]
\end{array}
$$

In a similar manner, the following formulas for $T_{k, m}^{b}(z), T_{k, m}^{c}(z), T_{k, m}^{d}(z)$ can be obtained respectively:

$$
\begin{aligned}
& T_{k, m}^{b}(z)=\frac{1}{2}\left(\frac{\overline{z_{0,0}^{k, m}}}{z_{0,0}^{k, m}}\right)^{2} \\
& \times\left[\frac{\left(z_{1,0}^{k, m}\right)^{2}}{2}-\frac{\left(z_{0,0}^{k, m}\right)^{2}}{2}+z z_{1,0}^{k, m}-z z_{0,0}^{k, m}+z^{2} \ln \left|\frac{z_{1,0}^{k, m}-z}{z_{0,0}^{k, m}-z}\right|\right]
\end{aligned}
$$




$$
\begin{aligned}
& +\frac{1}{2}\left(\overline{z_{1,1}^{k, m}} z_{1,1}^{k, m}\right)^{2}\left[\begin{array}{ll}
\frac{1}{z^{2}} & \left.\ln \frac{\left(z_{1,1}^{k, m}-z\right) z_{1,0}^{k, m}}{\left(z_{1,0}^{k, m}-z\right) z_{1,1}^{k, m}}+\frac{1}{z}\left(\frac{1}{z_{1,1}^{k, m}}-\frac{1}{z_{1,0}^{k, m}}\right)\right] \\
+\frac{1}{2}\left(\overline{z_{0,1}^{k, m}}\right. \\
z_{0,1}^{k, m}
\end{array}\right)^{2}\left[\frac{\left(z_{0,1}^{k, m}\right)^{2}}{2}-\frac{\left(z_{1,1}^{k, m}\right)^{2}}{2}+z z_{0,1}^{k, m}-z z_{1,1}^{k, m}+z^{2} \ln \frac{z_{0,1}^{k, m}-z}{z_{1,1}^{k, m}-z}\right] \\
& +\frac{1}{2}\left(\overline{z_{0,0}^{k, m}} z_{0,0}^{k, m}\right)^{2}\left[\frac{1}{z^{2}} \ln \frac{\left(z_{0,0}^{k, m}-z\right) z_{0,1}^{k, m}}{\left(z_{0,1}^{k, m}-z\right) z_{0,0}^{k, m}}+\frac{1}{z}\left(\frac{1}{z_{0,0}^{k, m}}-\frac{1}{z_{0,1}^{k, m}}\right)\right]
\end{aligned}
$$

$$
\begin{aligned}
& T_{k, m}^{c}(z)=\left(\left(\frac{\overline{\mathrm{z}_{0,0}^{k, m}}}{\mathrm{z}_{0,0}^{k, m}}\right)-\frac{1}{3}\left(\frac{\overline{\mathrm{z}_{0,0}^{k, m}}}{\mathrm{z}_{0,0}^{k, m}}\right)^{3}\right)\left[\frac{\left(z_{1,0}^{k, m}\right)^{3}}{3}-\frac{\left(z_{0,0}^{k, m}\right)^{3}}{3}+z \frac{\left(z_{1,0}^{k, m}\right)^{2}}{2}\right. \\
& \left.-z \frac{\left(z_{0,0}^{k, m}\right)^{2}}{2}+z^{2}\left(z_{1,0}^{k, m}-z_{0,0}^{k, m}\right)+z^{3} \ln \frac{z_{1,0}^{k, m}-z}{z_{0,0}^{k, m}-z}\right] \\
& +\left(z_{1,0}^{k, m} \overline{z_{1,0}^{k, m}}\right)\left\{z_{1,1}^{k, m}-z_{1,0}^{k, m}+z \ln \left(\frac{z_{1,1}^{k, m}-z}{z_{1,0}^{k, m}-z}\right)\right\}-\frac{\left(z_{1,0}^{k, m} \overline{z_{1,0}^{k, m}}\right)^{3}}{3} \\
& \times\left\{-\frac{1}{z^{3}} \cdot \ln \left(\frac{z_{1,1}^{k, m}}{z_{1,0}^{k, m}}\right)+\frac{1}{z^{2}} \cdot\left(\frac{1}{z_{1,1}^{k, m}}-\frac{1}{z_{1,0}^{k, m}}\right)\right. \\
& \left.+\frac{1}{2 z} \cdot\left(\frac{1}{\left(z_{1,1}^{k, m}\right)^{2}}-\frac{1}{\left(z_{1,0}^{k, m}\right)^{2}}\right)+\frac{1}{z^{3}} \cdot \ln \left(\frac{z_{1,1}^{k, m}-z}{z_{1,0}^{k, m}-z}\right)\right\} \\
& +\left(\left(\frac{\overline{\mathrm{z}_{0,1}^{k, m}}}{\mathrm{z}_{0,1}^{k, m}}\right)-\frac{1}{3}\left(\frac{\overline{\mathrm{z}_{0,1}^{k, m}}}{\mathrm{z}_{0,1}^{k, m}}\right)^{3}\right)\left[\frac{\left(z_{0,1}^{k, m}\right)^{3}}{3}-\frac{\left(z_{1,1}^{k, m}\right)^{3}}{3}+z \frac{\left(z_{0,1}^{k, m}\right)^{2}}{2}\right. \\
& \left.-z \frac{\left(z_{1,1}^{k, m}\right)^{2}}{2}+z^{2}\left(z_{0,1}^{k, m}-z_{1,1}^{k, m}\right)+z^{3} \ln \left|\frac{z_{0,1}^{k, m}-z}{z_{1,1}^{k, m}-z}\right|\right] \\
& +\left(z_{0,0}^{k, m} \overline{z_{0,0}^{k, m}}\right)\left\{z_{0,0}^{k, m}-z_{0,1}^{k, m}+z \ln \left(\frac{z_{0,0}^{k, m}-z}{z_{0,1}^{k, m}-z}\right)\right\}-\frac{\left(z_{0,0}^{k, m} \overline{z_{0,0}^{k, m}}\right)^{3}}{3} \\
& \times\left\{-\frac{1}{z^{3}} \cdot \ln \left(\frac{z_{0,0}^{k, m}}{z_{0,1}^{k, m}}\right)+\frac{1}{z^{2}} \cdot\left(\frac{1}{z_{0,0}^{k, m}}-\frac{1}{z_{0,1}^{k, m}}\right)\right. \\
& \left.+\frac{1}{2 z} \cdot\left(\frac{1}{\left(z_{0,0}^{k, m}\right)^{2}}-\frac{1}{\left(z_{0,1}^{k, m}\right)^{2}}\right)+\frac{1}{z^{3}} \cdot \ln \left(\frac{z_{0,0}^{k, m}-z}{z_{0,1}^{k, m}-z}\right)\right\} \text {, } \\
& T_{k, m}^{d}(z)=\frac{\overline{z_{0,0}^{k, m}}}{z_{0,0}^{k, m}}\left[z_{1,0}^{k, m}-z_{0,0}^{k, m}+z \ln \frac{z_{1,0}^{k, m}-z}{z_{0,0}^{k, m}-z}\right] \\
& +z_{1,1}^{k, m} \overline{z_{1,1}^{k, m}} \frac{1}{z} \ln \frac{\left(z_{1,1}^{k, m}-z\right) z_{1,0}^{k, m}}{\left(z_{1,0}^{k, m}-z\right) z_{1,1}^{k, m}} \\
& +\frac{\overline{z_{1,1}^{k, m}}}{z_{1,1}^{k, m}}\left[z_{0,1}^{k, m}-z_{1,1}^{k, m}+z \ln \frac{z_{0,1}^{k, m}-z}{z_{1,1}^{k, m}-z}\right]+\frac{z_{0,0}^{k, m} \overline{z_{0,0}^{k, m}}}{z} \ln \left(\frac{\left(z_{0,0}^{k, m}-z\right) z_{0,1}^{k, m}}{\left(z_{0,1}^{k, m}-z\right) z_{0,0}^{k, m}}\right) .
\end{aligned}
$$


Finally, we get an approximate formula

$$
\begin{gathered}
T(\rho \mid z) \approx \sum_{k=1}^{N-1} \sum_{m=0}^{M_{k}-1}\left(a_{k, m} T_{k, m}^{a}(z)+b_{k, m} T_{k, m}^{b}(z)\right. \\
\left.+c_{k, m} T_{k, m}^{c}(z)+d_{k, m} T_{k, m}^{d}(z)\right), z \in \Omega^{(2)}
\end{gathered}
$$

where coefficients $a_{k, m}, b_{k, m}, c_{k, m}, d_{k, m}$ and integrals $T_{k, m}^{a}, T_{k, m}^{b}, T_{k, m}^{c}$, and $T_{k, m}^{d}$ are defined by (5), (6), (7), (8) and (15),(16), (17), (18), respectively.

\section{Approximation of $S$}

In this section, we present quadrature formula for singular integral $S$. Applying spline approximation of $\rho$ in the domain $D_{k, m}$, we have

$$
\begin{aligned}
& S(\widehat{\rho} \mid z)=-\frac{1}{\pi} \sum_{k=1}^{N-1} \sum_{m=0}^{M_{k}-1}\left(a_{k, m} S_{k, m}^{a}(z)+b_{k, m} S_{k, m}^{b}(z)\right. \\
& \left.+c_{k, m} S_{k, m}^{c}(z)+d_{k, m} S_{k, m}^{d}(z)\right),
\end{aligned}
$$

where

$$
\begin{aligned}
& S_{k, m}^{a}(z)=-\frac{1}{\pi} \iint \frac{\zeta d \zeta}{D_{k, m}}, S_{k, m}^{b}(z)=-\frac{1}{\pi} \iint \frac{\bar{\zeta} d \zeta}{D_{k, m}}(\zeta-z)^{2} \\
& S_{k, m}^{c}(z)=-\frac{1}{\pi} \iint_{D_{k, m}} \frac{\left(\zeta^{2}-\bar{\zeta}^{2}\right) d \zeta}{(\zeta-z)^{2}}, S_{k, m}^{d}(z)=-\frac{1}{\pi} \iint_{D_{k, m}} \frac{d \zeta}{(\zeta-z)^{2}}
\end{aligned}
$$

From (1), it follows that

$$
\begin{aligned}
& S_{k, m}^{a}(z)=\frac{\partial T_{k, m}^{a}(z)}{\partial z}, S_{k, m}^{b}(z)=\frac{\partial T_{k, m}^{b}(z)}{\partial z}, \\
& S_{k, m}^{c}(z)=\frac{\partial T_{k, m}^{c}(z)}{\partial z}, S_{k, m}^{d}(z)=\frac{\partial T_{k, m}^{d}(z)}{\partial z} .
\end{aligned}
$$

Applying (15), (16), (17) and (18), we get

$$
\begin{aligned}
& S_{k, m}^{a}(z)=\frac{\overline{z_{0,0}^{k, m}}}{z_{0,0}^{k, m}} \\
& \times\left[z_{1,0}^{k, m}-z_{0,0}^{k, m}+2 z \ln \left(\frac{z_{1,0}^{k, m}-z}{z_{0,0}^{k, m}-z}\right)-z^{2}\left(\frac{1}{z_{1,0}^{k, m}-z}-\frac{1}{z_{0,0}^{k, m}-z}\right)\right] \\
& \left.+z_{1,0}^{k, m} \overline{z_{1,0}^{k, m}}\left[\frac{1}{z_{1,0}^{k, m}-z}-\frac{1}{z_{1,1}^{k, m}-z}\right]+z_{0,0}^{k, m} \frac{z_{0,0}^{k, m}}{z_{0,1}^{k, m}-z}-\frac{1}{z_{0,0}^{k, m}-z}\right] \\
& \quad+\frac{z_{1,1}^{k, m}}{z_{1,1}^{k, m}}\left[z_{0,1}^{k, m}-z_{1,1}^{k, m}+2 z \ln \left(\frac{z_{0,1}^{k, m}-z}{z_{1,1}^{k, m}-z}\right)-z^{2}\left(\frac{1}{z_{0,1}^{k, m}-z}-\frac{1}{z_{1,1}^{k, m}-z}\right)\right],
\end{aligned}
$$




$$
\begin{aligned}
& S_{k, m}^{b}(z)=\frac{1}{2}\left(\frac{\overline{z_{0,0}^{k, m}}}{z_{0,0}^{k, m}}\right)^{2} \\
& \times\left[z_{1,0}^{k, m}-z_{0,0}^{k, m}+2 z \ln \left(\frac{z_{1,0}^{k, m}-z}{z_{0,0}^{k, m}-z}\right)+z^{2}\left(\frac{1}{z_{0,0}^{k, m}-z}-\frac{1}{z_{1,0}^{k, m}-z}\right)\right] \\
& +\frac{1}{2}\left(\overline{z_{1,1}^{k, m}} z_{1,1}^{k, m}\right)^{2}\left[-\frac{2}{z^{3}} \ln \frac{\left(z_{1,1}^{k, m}-z\right) z_{1,0}^{k, m}}{\left(z_{1,0}^{k, m}-z\right) z_{1,1}^{k, m}}+\frac{1}{z^{2}}\left(\frac{1}{z_{1,0}^{k, m}-z}-\frac{1}{z_{1,1}^{k, m}-z}\right)\right. \\
& \left.-\frac{1}{z^{2}}\left(\frac{1}{z_{1,1}^{k, m}}-\frac{1}{z_{1,0}^{k, m}}\right)\right]+\frac{1}{2}\left(\frac{\overline{z_{0,1}^{k, m}}}{z_{0,1}^{k, m}}\right)^{2} \\
& \times\left[z_{0,1}^{k, m}-z_{1,1}^{k, m}+2 z \ln \left(\frac{z_{0,1}^{k, m}-z}{z_{1,1}^{k, m}-z}\right)+z^{2}\left(\frac{1}{z_{1,1}^{k, m}-z}-\frac{1}{z_{0,1}^{k, m}-z}\right)\right] \\
& +\frac{1}{2}\left(\overline{z_{0,0}^{k, m}} z_{0,0}^{k, m}\right)^{2}\left[-\frac{2}{z^{3}} \ln \frac{\left(z_{0,0}^{k, m}-z\right) z_{0,1}^{k, m}}{\left(z_{0,1}^{k, m}-z\right) z_{0,0}^{k, m}}+\frac{1}{z^{2}}\left(\frac{1}{z_{0,1}^{k, m}-z}-\frac{1}{z_{0,0}^{k, m}-z}\right)\right. \\
& \left.-\frac{1}{z^{2}}\left(\frac{1}{z_{0,0}^{k, m}}-\frac{1}{z_{0,1}^{k, m}}\right)\right] \text {, } \\
& S_{k, m}^{c}(z)=\left(\left(\frac{\overline{\mathrm{z}_{0,0}^{k, m}}}{\mathrm{z}_{0,0}^{k, m}}\right)-\frac{1}{3}\left(\frac{\overline{\mathrm{z}_{0,0}^{k, m}}}{\mathrm{z}_{0,0}^{k, m}}\right)^{3}\right)\left[\frac{\left(z_{1,0}^{k, m}\right)^{2}}{2}-\frac{\left(z_{0,0}^{k, m}\right)^{2}}{2}\right. \\
& \left.+2 z\left(z_{1,0}^{k, m}-z_{0,0}^{k, m}\right)+3 z^{2} \ln \frac{z_{1,0}^{k, m}-z}{z_{0,0}^{k, m}-z}+z^{3}\left(\frac{1}{z_{0,0}^{k, m}-z}-\frac{1}{z_{1,0}^{k, m}-z}\right)\right] \\
& +\left(z_{1,0}^{k, m} \overline{z_{1,0}^{k, m}}\right)\left\{\ln \left(\frac{z_{1,1}^{k, m}-z}{z_{1,0}^{k, m}-z}\right)+z\left(\frac{1}{z_{1,0}^{k, m}-z}-\frac{1}{z_{1,1}^{k, m}-z}\right)\right\}-\frac{\left(z_{1,0}^{k, m} \overline{z_{1,0}^{k, m}}\right)^{3}}{3} \\
& \times\left\{\frac{3}{z^{4}} \cdot \ln \left(\frac{z_{1,1}^{k, m}}{z_{1,0}^{k, m}}\right)-\frac{2}{z^{3}} \cdot\left(\frac{1}{z_{1,1}^{k, m}}-\frac{1}{z_{1,0}^{k, m}}\right)-\frac{1}{2 z^{2}} \cdot\left(\frac{1}{\left(z_{1,1}^{k, m}\right)^{2}}-\frac{1}{\left(z_{1,0}^{k, m}\right)^{2}}\right)\right. \\
& \left.-\frac{3}{z^{4}} \cdot \ln \left(\frac{z_{1,1}^{k, m}-z}{z_{1,0}^{k, m}-z}\right)+\frac{1}{z^{3}} \cdot\left(\frac{1}{z_{1,0}^{k, m}-z}-\frac{1}{z_{1,1}^{k, m}-z}\right)\right\} \\
& +\left(\left(\frac{\overline{\mathrm{z}_{0,1}^{k, m}}}{\mathrm{z}_{0,1}^{k, m}}\right)-\frac{1}{3}\left(\frac{\overline{\mathrm{z}_{0,1}^{k, m}}}{\mathrm{z}_{0,1}^{k, m}}\right)^{3}\right)\left[\frac{\left(z_{0,1}^{k, m}\right)^{2}}{2}-\frac{\left(z_{1,1}^{k, m}\right)^{2}}{2}+2 z\left(z_{0,1}^{k, m}-z_{1,1}^{k, m}\right)\right. \\
& \left.+3 z^{2} \ln \left(\frac{z_{0,1}^{k, m}-z}{z_{1,1}^{k, m}-z}\right)+z^{3}\left(\frac{1}{z_{1,1}^{k, m}-z}-\frac{1}{z_{0,1}^{k, m}-z}\right)\right] \\
& +\left(z_{0,0}^{k, m} \overline{z_{0,0}^{k, m}}\right)\left\{\ln \left(\frac{z_{0,0}^{k, m}-z}{z_{0,1}^{k, m}-z}\right)+z\left(\frac{1}{z_{0,1}^{k, m}-z}-\frac{1}{z_{0,0}^{k, m}-z}\right)\right\}-\frac{\left(z_{0,0}^{k, m} \overline{z_{0,0}^{k, m}}\right)^{3}}{3} \\
& \times\left\{\frac{3}{z^{4}} \cdot \ln \left(\frac{z_{0,0}^{k, m}}{z_{0,1}^{k, m}}\right)-\frac{2}{z^{3}} \cdot\left(\frac{1}{z_{0,0}^{k, m}}-\frac{1}{z_{0,1}^{k, m}}\right)-\frac{1}{2 z^{2}} \cdot\left(\frac{1}{\left(z_{0,0}^{k, m}\right)^{2}}-\frac{1}{\left(z_{0,1}^{k, m}\right)^{2}}\right)\right. \\
& \left.-\frac{3}{z^{4}} \cdot \ln \left(\frac{z_{0,0}^{k, m}-z}{z_{0,1}^{k, m}-z}\right)+\frac{1}{z^{3}} \cdot\left(\frac{1}{z_{0,1}^{k, m}-z}-\frac{1}{z_{0,0}^{k, m}-z}\right)\right\},
\end{aligned}
$$




$$
\begin{aligned}
& S_{k, m}^{d}(z)=\frac{\overline{z_{0,0}^{k, m}}}{z_{0,0}^{k, m}}\left[\ln \frac{z_{1,0}^{k, m}-z}{z_{0,0}^{k, m}-z}+z\left(\frac{1}{z_{0,0}^{k, m}-z}-\frac{1}{z_{1,0}^{k, m}-z}\right)\right] \\
& -z_{1,1}^{k, m} \overline{z_{1,1}^{k, m}} \frac{1}{z^{2}} \ln \frac{\left(z_{1,1}^{k, m}-z\right) z_{1,0}^{k, m}}{\left(z_{1,0}^{k, m}-z\right) z_{1,1}^{k, m}}+z_{1,1}^{k, m} z_{1,1}^{k, m} \frac{1}{z}\left(\frac{1}{z_{1,0}^{k, m}-z}-\frac{1}{z_{1,1}^{k, m}-z}\right) \\
& +\frac{\overline{z_{1,1}^{k, m}}}{z_{1,1}^{k, m}}\left[\ln \frac{z_{0,1}^{k, m}-z}{z_{1,1}^{k, m}-z}+z\left(\frac{1}{z_{1,1}^{k, m}-z}-\frac{1}{z_{0,1}^{k, m}-z}\right)\right] \\
& -\frac{z_{0,0}^{k, m}}{z_{0,0}^{k, m}} \ln \left(\frac{\left(z_{0,0}^{k, m}-z\right) z_{0,1}^{k, m}}{\left(z_{0,1}^{k, m}-z\right) z_{0,0}^{k, m}}\right)+\frac{z_{0,0}^{k, m} \overline{z_{0,0}^{k, m}}}{z}\left(\frac{1}{z_{0,1}^{k, m}-z}-\frac{1}{z_{0,0}^{k, m}-z}\right) .
\end{aligned}
$$

Therefore, we obtain quadrature formula

$$
\begin{gathered}
S(\rho \mid z) \approx \sum_{k=1}^{N-1} \sum_{m=0}^{M_{k}-1}\left(a_{k, m} S_{k, m}^{a}(z)+b_{k, m} S_{k, m}^{b}(z)\right. \\
\left.+c_{k, m} S_{k, m}^{c}(z)+d_{k, m} S_{k, m}^{d}(z)\right), z \in \Omega^{(2)}
\end{gathered}
$$

where coefficients $a_{k, m}, b_{k, m}, c_{k, m}$, and $d_{k, m}$ and integrals $S_{k, m}^{a}, S_{k, m}^{b}, S_{k, m}^{c}$ and $S_{k, m}^{d}$ are defined by (5), (6), (7), (8) and (19), (20), (21), (22), respectively.

\section{Error analysis}

In this section, we present numerical results of error for proposed approximate formulas of $T$ and $S$ in test examples by using MATLAB program.

Let us take $l_{2}\left(\Omega^{(j)}\right)(j=1,2)$, the spaces of the grid functions $\rho^{\tau, h}$, defined on $\Omega^{(j)}$ equipped with the norm

$$
\left\|\rho^{\tau, h}\right\|_{l_{2}\left(\Omega^{(j)}\right)}=\sqrt{\pi} \tau\left[\sum_{k=1}^{N-1} \sum_{m=1}^{M_{k}-1}\left|\rho\left(r_{k} e^{i \theta_{k, m}}\right)\right|^{2}\right]^{1 / 2},
$$

where $\rho_{k, m}=\rho\left(r_{k} e^{i \theta_{k, m}}\right), r_{k} e^{i \theta_{k, m}} \in \Omega^{(j)}$.

Table 1 displays the value of error of $T(\rho \mid z)$ defined by

$$
E r T=\sqrt{\pi} \tau\left[\sum_{k=1}^{N-1} \sum_{m=1}^{M_{k}-1}\left|T\left(\rho \mid z_{0,0}^{* k, m}\right)-T\left(\widehat{\rho} \mid z_{0,0}^{* k, m}\right)\right|^{2}\right]^{1 / 2}
$$

for $N=5,10,20,40$. 
Table 1. Error analysis for $T(\rho \mid z)$

\begin{tabular}{|l|l|l|l|l|l|l|}
\hline & $\rho(z)$ & $T(\rho \mid z)$ & $N=5$ & $N=10$ & $N=20$ & $N=40$ \\
\hline 1 & $z \bar{z}$ & $\frac{z \bar{z}^{2}}{2}$ & 0.2450 & 0.0278 & 0.0059 & 0.0014 \\
\hline 2 & $z \bar{z}^{2}$ & $\frac{z \bar{z}^{3}}{3}$ & 0.1803 & 0.0162 & 0.0038 & $9.4 \times 10^{-04}$ \\
\hline 3 & $z \bar{z}^{3}$ & $\frac{z \bar{z}^{4}}{4}$ & 0.1611 & 0.0338 & 0.0076 & 0.0019 \\
\hline 4 & $z$ & $z \bar{z}-1$ & 0.3018 & 0.0377 & 0.0047 & $5.8 \times 10^{-04}$ \\
\hline 5 & $\bar{z}$ & $\frac{\bar{z}^{2}}{2}$ & 0.0989 & 0.0124 & 0.0015 & $1.9 \times 10^{-04}$ \\
\hline 6 & $z^{2}$ & $z^{2} \bar{z}-z$ & 0.1902 & 0.0227 & 0.0048 & 0.0012 \\
\hline 7 & $\bar{z}^{2}$ & $\frac{\bar{z}^{3}}{3}$ & 0.1489 & 0.0206 & 0.0047 & 0.0012 \\
\hline 8 & $\bar{z}^{3}$ & $\frac{\bar{z}^{4}}{4}$ & 0.2027 & 0.0526 & 0.0133 & 0.0033 \\
\hline 9 & $z^{2} \bar{z}$ & $\frac{z^{2} \bar{z}^{2}}{2}-\frac{1}{2}$ & 0.1112 & 0.0226 & 0.0059 & 0.0015 \\
\hline 10 & $z^{2} \bar{z}^{2}$ & $\frac{z^{2} \bar{z}^{3}}{3}$ & 0.1417 & 0.0194 & 0.0048 & 0.0012 \\
\hline 11 & $z^{2} \bar{z}^{3}$ & $\frac{z^{2} \bar{z}^{4}}{4}$ & 0.1215 & 0.0212 & 0.0049 & 0.0012 \\
\hline 12 & $z^{3} \bar{z}$ & $\frac{z^{3} \bar{z}^{2}}{2}-\frac{z}{2}$ & 0.1369 & 0.0279 & 0.0061 & 0.0015 \\
\hline 13 & $z^{3} \bar{z}^{2}$ & $\frac{z^{3} \bar{z}^{3}}{3}-\frac{1}{3}$ & 0.1144 & 0.0344 & 0.0086 & 0.0022 \\
\hline 14 & $z^{3} \bar{z}^{3}$ & $\frac{z^{3} \bar{z}^{4}}{4}$ & 0.0885 & 0.0181 & 0.0045 & 0.0011 \\
\hline 15 & $z^{3} \bar{z}^{4}$ & $\frac{z^{3} \bar{z}^{5}}{5}$ & 0.1096 & 0.0254 & 0.0057 & 0.0014 \\
\hline 16 & $z^{3}$ & $z^{3} \bar{z}-z^{2}$ & 0.1807 & 0.0388 & 0.0096 & 0.0024 \\
\hline 17 & $z^{2}-\bar{z}^{2}$ & $z^{2} \bar{z}-z-\frac{z^{3}}{3}$ & 0.1785 & 0.0112 & $6.9 \times 10^{-04}$ & $4.3 \times 10^{-05}$ \\
\hline
\end{tabular}

Table 2 layouts the value of error of $S(\rho \mid z)$ defined by

$$
\operatorname{Er} S=\sqrt{\pi} \tau\left[\sum_{k=1}^{N-1} \sum_{m=1}^{M_{k}-1}\left|S\left(\rho \mid z_{0,0}^{* k, m}\right)-S\left(\widehat{\rho} \mid z_{0,0}^{* k, m}\right)\right|^{2}\right]^{1 / 2}
$$

for $N=5,10,20,40$.

\section{Conclusion}

In this paper, we construct second-order of accuracy quadrature formulas for the numerical calculation of the Vekua types two-dimensional potential and singular integral operators in the unit disk of complex plane. We propose quadrature formulas for these integrals which based on first-order spline approximation of twodimensional function. MATLAB program are used for numerical experiments in test examples. 
Table 2. Error analysis for $S(\rho \mid z)$

\begin{tabular}{|l|l|l|l|l|l|l|}
\hline & $\rho(z)$ & $S(\rho \mid z)$ & $N=5$ & $N=10$ & $N=20$ & $N=40$ \\
\hline 1 & $z \bar{z}$ & $\frac{\bar{z}^{2}}{2}$ & 0.0775 & 0.0138 & 0.0030 & $7.1 \times 10^{-04}$ \\
\hline 2 & $z \bar{z}^{2}$ & $\frac{\bar{z}^{3}}{3}$ & 0.4025 & 0.0683 & 0.0230 & 0.0083 \\
\hline 3 & $z \bar{z}^{3}$ & $\frac{\bar{z}^{4}}{4}$ & 0.6517 & 0.1549 & 0.0567 & 0.0207 \\
\hline 4 & $z$ & $\bar{z}$ & $8.7 \times 10^{-15}$ & $1.5 \times 10^{-13}$ & $2.1 \times 10^{-12}$ & $5.1 \times 10^{-11}$ \\
\hline 5 & $\bar{z}$ & 0 & 0.3072 & 0.0768 & 0.0192 & 0.0048 \\
\hline 6 & $z^{2}$ & $2 z \bar{z}-1$ & 0.6155 & 0.1169 & 0.0324 & 0.0110 \\
\hline 7 & $\bar{z}^{2}$ & 0 & 0.6525 & 0.1174 & 0.0322 & 0.0110 \\
\hline 8 & $\bar{z}^{3}$ & 0 & 0.7794 & 0.1901 & 0.0685 & 0.0249 \\
\hline 9 & $z^{2} \bar{z}$ & $z \bar{z}^{2}$ & 0.4335 & 0.0724 & 0.0239 & 0.0085 \\
\hline 10 & $z^{2} \bar{z}^{2}$ & $\frac{2}{3} z \bar{z}^{3}$ & 0.0985 & 0.0261 & 0.0067 & 0.0017 \\
\hline 11 & $z^{2} \bar{z}^{3}$ & $\frac{1}{2} z \bar{z}^{4}$ & 0.3466 & 0.0906 & 0.0332 & 0.0121 \\
\hline 12 & $z^{3} \bar{z}$ & $\frac{3}{2} z^{2} \bar{z}^{2}-\frac{1}{2}$ & 0.5976 & 0.1478 & 0.0557 & 0.0206 \\
\hline 13 & $z^{3} \bar{z}^{2}$ & $z^{2} \bar{z}^{3}$ & 0.3912 & 0.0981 & 0.0346 & 0.0124 \\
\hline 14 & $z^{3} \bar{z}^{3}$ & $\frac{3}{4} z^{2} \bar{z}^{4}$ & 0.1748 & 0.0463 & 0.0118 & 0.0030 \\
\hline 15 & $z^{3} \bar{z}^{4}$ & $\frac{3}{5} z^{2} \bar{z}^{5}$ & 0.3654 & 0.1114 & 0.0407 & 0.0150 \\
\hline 16 & $z^{3}$ & $3 z^{2} \bar{z}-2 z$ & 0.8176 & 0.1897 & 0.0682 & 0.0248 \\
\hline 17 & $z^{2}-\bar{z}^{2}$ & $2 z \bar{z}-1$ & 0.7305 & 0.0913 & 0.0114 & 0.0014 \\
\hline
\end{tabular}

\section{Bibliography}

[1] E. Anderes and M. A. Coram, Two-dimensional density estimation using smooth invertible transformation, J. Stat. Plan. Infer. 141(3) (2011), 1183-1193.

[2] C. Ashyralyev, Numerical Algorithms of the Solution for Singular Integral Equations and Their Applications in Hydrodynamics, Ylym, Ashgabat, 1994.

[3] C. Ashyralyev and Z. Cakir, Approximate solution of the two-dimensional singular integral equation, AIP Conference Proceedings 1611 (2014), 73-77.

[4] C. Ashyralyyev and B. Oztürk, Some approximations of second order derivatives complex-valued function, Bulletin of the Karaganda University-Mathematics 91(3) (2018), 17-23.

[5] C. Ashyralyev and V. N. Monakhov, Iterative algorithm for solving two-dimensional singular integral equations, Din. Sploshnoi Sredy 101 (1991), 21-29.

[6] F. Gakhov, Boundary Value Problems, Courier Dover Publications, 1990.

[7] P. Daripa and D. Mashat, Singular integral transforms and fast numerical algorithms, Numerical Algorithms 18 (1998), 133-157. 
[8] V. D. Didenko and B. Silbermann, On the approximate solution of some twodimensional singular integral equations, Math. Meth. Appl. Sci. 24 (2001), 11251138 .

[9] A. S. Ismail, On the numerical solution of two-dimensional singular integral equation, Applied Mathematics and Computation 173(1) (2006), 389-393.

[10] V. Monakhov, Boundary-Value Problems with Free Boundaries for Elliptic Systems of Equations, Translations of Mathematical Monographs (AMS), 1983.

[11] N. I. Muskhelishvili, Singular Integral Equations, Noordhoff International Publishing, Groningen, 1953.

[12] S. V. Rogosin, On nonlinear Vekua type equations, Nonlinear Analysis: Modeling and Control 11 (2006), 187-200.

[13] I. N. Vekua, Generalized Analytic Functions, Pergamon Press, Oxford, 1962.

[14] Yu. S. Zavyalov, B. I. Kvasov and V. L. Miroshnichenko, Methods of Spline Functions, Nauka, Moscow, 1980 (in Russian).

Received July 31, 2019; revised November 29, 2019; accepted December 9, 2019.

\section{Author information}

Charyyar Ashyralyyev, Department of Mathematical Engineering, Gumushane University, 29100, Gumushane, Turkey.

E-mail: charyyar@gumushane.edu.tr

Sedanur Efe, Department of Mathematical Engineering, Gumushane University, 29100, Gumushane, Turkey.

E-mail: sedanurefe06@gmail.com 